\title{
miR-204 inhibits the proliferation and invasion of renal cell carcinoma by inhibiting RAB22A expression
}

\author{
FENG XIONG ${ }^{1,2}$, KEYUN LIU $^{1,2}$, FUMEI ZHANG $^{1,2}$, KAIHUI SHA $^{1,2}$, XINYUAN WANG $^{1,2}$, \\ XIAOJUAN GUO ${ }^{1,2}$ and NING HUANG ${ }^{1,2}$ \\ ${ }^{1}$ Research Unit of Infection and Immunity, and ${ }^{2}$ Department of Pathophysiology, West China College \\ of Basic and Forensic Medicine, Sichuan University, Chengdu, Sichuan 610041, P.R. China
}

Received October 17, 2015; Accepted January 7, 2016

DOI: $10.3892 /$ or.2016.4624

\begin{abstract}
While miR-204 expression may be linked to renal cell carcinoma (RCC) progression, the detailed mechanisms remain unclear. In the present study, we demonstrated that miR-204 was differentially expressed in RCC tissues when compared with surrounding normal kidney tissues. Ectopic overexpression of miR-204 in human RCC cells suppressed cell proliferation and invasion in vitro and in vivo. Mechanism dissection revealed that miR-204 may function through RAB22A signals to inhibit RCC proliferation and invasion. Overexpression of RAB22A by oe-RAB22A was able to partially reverse the miR-204-mediated suppression of RCC tumor progression. Together, these results revealed that miR-204 suppressed RCC proliferation and invasion by directly targeting the RAB22A gene. Targeting newly identified RAB22A with miR-204 may aid in the suppression of $\mathrm{RCC}$ proliferation and invasion.
\end{abstract}

\section{Introduction}

Renal cell carcinoma (RCC) is the most common type of kidney tumor and accounts for nearly $80 \%$ of all renal cancer cases in adults (1). It is described as one of the most lethal urological cancers worldwide (2). Although various small renal tumors can be detected at an early stage, one in 3 RCC tumors is detected at a later metastatic stage (3). These late-stage tumors are associated with a poor prognosis due to the fact that metastatic RCC is highly resistant to many therapies including radiotherapy and chemotherapy $(4,5)$. Although recently developed targeted therapies for advanced RCC have achieved certain improvement in the treatment of selective patients, the majority of advanced RCC patients remain refractory to treatment $(6,7)$. Thus, understanding the molecular mechanisms

Correspondence to: Dr Ning Huang, Research Unit of Infection and Immunity, West China College of Basic and Forensic Medicine, Sichuan University, 17 Third Section, Ren Min Nan Road, Chengdu, Sichuan 610041, P.R. China

E-mail: huangning2015@sina.com

Key words: miR-204, renal cell carcinoma, RAB22A, proliferation, invasion involved in RCC progression is important in order to identify new targets for novel and more effective therapy.

Emerging evidence shows that microRNAs (miRNAs), a group of small non-coding RNAs of 22 nucleotides in length, negatively regulate gene expression, primarily by targeting the 3'-untranslated region (3'-UTR) of messenger RNAs (mRNAs) $(8,9)$. miRNAs are known to contribute to tumorigenesis in a wide spectrum of human cancers, including RCC (10). Recent studies have demonstrated the regulatory functions of miRNAs in RCC cell proliferation (11-13), apoptosis $(14,15)$, invasion and metastasis (16-18). A predominant and systemic alteration in miRNA expression during renal carcinogenesis has been indicated by studies of miRNA expression profiling (16).

miR-204 is an miRNA which is enriched in germline and mesoderm-derived tissues, such as the testis, uterus, kidney, lung, heart and bladder (19). Numerous studies have shown that miR-204 is downregulated and acts as a tumor suppressor in various human malignancies, including head and neck (20), gastric (21) and pancreatic cancer (22). Recent studies indicate that miR-204 is downregulated in RCC and is a critical regulator of tumor growth via inhibition of autophagy (23), implying the potential role of miR-204 in renal tumors. However, the exact function of miR-204 in RCC remains elusive.

In the present study, we identified miR-204 as one of the most significantly downregulated miRNAs in RCC tissues and cells and a critical suppressor of RCC cell proliferation and invasion both in vitro and in vivo. We further demonstrated that attenuation of miR-204 expression in RCC is associated with RAB22A as a potential candidate target gene. In addition, our data based on clinical RCC tissues indicated that miR-204 may be a potential biomarker for discriminating between RCC and normal tissues.

\section{Materials and methods}

Human samples. Surgical specimens (paired normal and cancerous tissues) were obtained from 65 patients with kidney tumors at the Department of Urology, Chengdu Third People Hospital and were freshly frozen in liquid nitrogen until use. Informed consent was obtained from the patients, and the study was approved by the Institutional Review Board of Sichuan University. 
Table I. Primers used for real-time PCR and vector construction.

\begin{tabular}{llc}
\hline Primer names & & Sequences \\
\hline miR-204 & & 5'-UUCCCUUUGUCAUCCUAUGCCU-3' \\
RAB22A & F & 5'-TTGTAGTTGCCATTGCAGGA-3' \\
& R & 5'-AGGCTGTCTTCGGAGTTTGA-3' \\
GAPDH & F & 5'-GGAGCGAGATCCCTCCAAAAT-3' \\
& R & 5'-GGCTGTTGTCATACTTCTCATGG-3' \\
miR-204 inhibitor & & GGCUACAGUCUUUCUUCAUGUGACUCGUGGACUUCCCUUU \\
oe-miR-204 & F & 5'-CCTTAATTAAGAAGGCAAAGGGACGTTCAA-3' \\
& R & 5'-CCGGCGCGCCGTTTAAACTTCATCCAAGCAGCTCTGGA-3' \\
oe-RAB22A & F & 5'-CCTTAATTAAATGGCGCTGAGGGAGCTCAAAGTGTGTC-3' \\
& R & 5'-CCGGCGCGCCGTTTAAACTCAGCAGCAGCTCCGCTTTGGCTCTGA-3'
\end{tabular}

F, forward; R, reverse.

Cell culture and transfection. The human RCC cell lines, OSRC-2, 786-O, ACHN, A498 and 769P, and the primary renal tubular cell line HK2 were purchased from the American Type Culture Collection (ATCC; Manassas, VA, USA). The OSRC-2, 786-O, ACHN, A498, 769P and HK2 cells were cultured in Dulbecco's modified Eagle's medium (DMEM) supplemented with $10 \%$ fetal bovine serum (FBS) in a humidified $5 \% \mathrm{CO}_{2}$ environment at $37^{\circ} \mathrm{C}$.

The miRNA-204 expression plasmid was generated by cloning the genomic pre-miR-204 gene, with a 200-bp sequence on each flanking side, into retroviral transfer plasmid pWPI to generate the plasmid pWPI-miR-204.

To generate RAB22A-overexpressing stable clones, OSRC-2 and 786-O cells were transfected with lentiviral vectors, $\mathrm{pWPI}$ RAB22A/pWPI-Vec, with the PAX2 packaging plasmid and PMD2G envelope plasmid. Then, these were transfected into OSRC-2 and 786-O cells for $48 \mathrm{~h}$ to obtain a lentivirus soup. The lentivirus soup was collected and frozen at $-80^{\circ} \mathrm{C}$ for use. Anti-miR-204 or negative control inhibitors (RiboBio Co., Ltd.) were transfected into confluent cells with Lipofectamine 3000 (Life Technologies, Inc.). To obtain stably infected OSRC-2 and $786-\mathrm{O}$ cells, the cells were cultured to $\sim 70 \%$ of the plates, and then added by a concentration of $1.0 \times 10^{4}$ cells with miR-204 and mock or anti-miR-204 and anti-miR-NC.

Real-time RT-PCR assays. The miRNA RT-PCR assays were performed using the TaqMan miRNA reverse transcription kit and the 7500 Fast Real-Time PCR system (both from Applied Biosystems) for quantitative miRNA detection. Each miRNA TaqMan PCR probe was purchased from Applied Biosystems. The real-time RT-PCR assays were performed using the 7500 Fast Real-Time PCR system for quantitative mRNA detection and with iTaq Fast SYBR-Green Supermix (Bio-Rad). The primers used for real-time PCR and vector construction are listed in Table I.

Western blot analysis. Cells were lysed in RIPA buffer and proteins $(20 \mu \mathrm{g})$ were separated on $8-10 \%$ SDS/PAGE gel and then transferred onto PVDF membranes (Millipore, Billerica, MA, USA). After blocking the membranes, they were incubated with appropriate dilutions of specific primary antibodies. The blots were then incubated with HRP-conjugated secondary antibodies and visualized using an ECL system (Thermo Fisher Scientific, Rochester, NY, USA). Immunoblots were performed with primary monoclonal antibodies RAB22A (Abcam, Cambridge, UK) and GAPDH (Sigma, USA).

$R C C$ cell invasion assay. The invasion capability of RCC cells was determined by the Transwell assay. Before seeding the cells, $10 \mathrm{ml}$ of Matrigel (BD, Inc.) was dissolved in $50 \mathrm{ml}$ serum-free DMEM, applied to the upper chamber of 8-mm pore-size polycarbonate membrane filters (Corning, Inc., Corning, NY, USA), and put into an incubator for $5 \mathrm{~h}$. RCC cells were then harvested and seeded with serum-free DMEM into the upper chamber at $1 \times 10^{5}$ cells/well, and the bottom chamber of the apparatus contained DMEM with $10 \%$ FBS. The Transwells were then incubated for $48 \mathrm{~h}$ at $37^{\circ} \mathrm{C}$. Following incubation, the invaded cells that had attached to the lower surface of the membrane were fixed with $4 \%$ paraformaldehyde and stained with $1 \%$ toluidine blue. Cell numbers were counted in five randomly chosen microscopic fields (magnification, x100) per membrane.

RCC cell proliferation assay. RCC cells were seeded in 24-well plates (3,000 cells/well) and cultured for 24, 48, 72 and $96 \mathrm{~h}$. Cells were harvested, and the cell number was calculated using MTT agent. DMSO was used as the control.

Luciferase assay. Cells were cultured in 24-well plates and transfected with $0.2 \mathrm{mg}$ of either wild-type or mutant 3'-UTR of RAB22A. The plasmid contained firefly luciferase. After $48 \mathrm{~h}$ of transfection, the cells were lysed with $1 \mathrm{X}$ reporter lysis buffer and firefly and Renilla luciferase activities were measured using the Dual Luciferase Reporter Assay (Promega) according to the manufacturer's instructions. Firefly luciferase activity was standardized to the Renilla activity as a control. All transfection experiments were conducted in triplicate and repeated 3 times independently.

In vivo metastasis studies. Male 6- to 8-week-old nude mice were purchased from NCI. Sixteen mice were divided into 
Table II. The relative miR-204 expression levels in the RCC samples.

\begin{tabular}{|c|c|c|c|c|}
\hline \multirow[b]{2}{*}{ Parameters } & \multirow[b]{2}{*}{$\mathrm{N}$} & \multicolumn{2}{|c|}{ miR-204 expression } & \multirow[b]{2}{*}{ P-value } \\
\hline & & High & Low & \\
\hline \multicolumn{5}{|l|}{ Gender } \\
\hline Male & 40 & 18 & 22 & \multirow[t]{2}{*}{0.128} \\
\hline Female & 25 & 12 & 13 & \\
\hline \multicolumn{5}{|l|}{ Age (years) } \\
\hline$<60$ & 35 & 15 & 20 & \multirow[t]{2}{*}{0.212} \\
\hline$\geq 60$ & 30 & 12 & 18 & \\
\hline \multicolumn{5}{|c|}{ Tumor size $(\mathrm{cm})$} \\
\hline$\leq 4$ & 16 & 10 & 6 & \multirow[t]{3}{*}{0.191} \\
\hline$>4$ and $\leq 7$ & 28 & 13 & 15 & \\
\hline$>7$ & 21 & 10 & 11 & \\
\hline \multicolumn{5}{|l|}{ pT stage } \\
\hline $\mathrm{T} 1-2$ & 42 & 18 & 24 & \multirow[t]{2}{*}{$0.036^{\mathrm{a}}$} \\
\hline T3-4 & 23 & 15 & 8 & \\
\hline \multicolumn{5}{|l|}{ Fuhrman } \\
\hline I-II & 41 & 25 & 16 & \multirow[t]{2}{*}{$0.042^{\mathrm{a}}$} \\
\hline III-IV & 24 & 16 & 8 & \\
\hline \multicolumn{5}{|l|}{ Tumor side } \\
\hline Left & 30 & 15 & 15 & \multirow[t]{2}{*}{0.345} \\
\hline Right & 35 & 19 & 16 & \\
\hline \multicolumn{5}{|l|}{ Metastasis } \\
\hline Positive & 48 & 14 & 34 & \multirow[t]{2}{*}{$0.023^{\mathrm{a}}$} \\
\hline Negative & 17 & 5 & 12 & \\
\hline
\end{tabular}

RCC, renal cell carcinoma. ${ }^{a} \mathrm{P}<0.05$, indicating statistical significance.

2 groups $(\mathrm{n}=8)$. The first group of mice was injected into the renal capsule with $1 \times 10^{6}$ oe-miR-204 OSRC-2 cells (mixture with Matrigel, 1:1); the second group of mice was injected with $1 \times 10^{6}$ miR-NC OSRC- 2 cells. Metastasis in mice was assessed using a fluorescent imager (IVIS Spectrum; Caliper Life Sciences, Hopkinton, MA, USA) at 4 different time points $(1,4,5$ and 6 weeks after injection). After monitoring with the imager, the mice were sacrificed and the metastatic sites were further examined.

Statistical analysis. Data are expressed as the mean \pm SEM from at least 3 independent experiments. Statistical analyses were carried out with a paired t-test using SPSS 17.0 (SPSS, Inc., Chicago, IL, USA). In the in vivo study, measurements of tumor metastasis among the 3 groups were analyzed by one-way ANOVA coupled with the Newman-Keuls test. $\mathrm{P}<0.05$ was considered to indicate a statistically significant result.

\section{Results}

miR-204 is downregulated in RCC tissues and cell lines. We firstly analyzed the expression of miR-204 in 65 human RCC tissues with paired adjacent normal tissues by real-time PCR.
Consistent with the statistical result of Wotschofsky et al from GSE37989 in GEO database (24) (Fig. 1A), real-time PCR indicated that miR-204 expression was markedly downregulated in $90 \%(59 / 65)$ of the RCC tissues $(\mathrm{P}<0.0001$; Fig. 1B and C). Although the miR-204 mRNA level was not associated with tumor side, its expression was found to be gradually decreased during tumor progression (Table II). Notably, miR-204 was lowly expressed in RCC cell lines, including A498, OSRC-2, 769P, ACHN and OSRC-2 cells when compared with its expression level in the HK2 cell line (Fig. 1D). The above results revealed that miR-204 is downregulated in RCC, in keeping with the previous study that the miR-204 level was downregulated in RCC compared with matched normal renal tissues (23).

miR-204 attenuates RCC cell proliferation and invasion in vitro. Previous research found that miR-204 suppressed RCC proliferation via inhibition of LC3B-mediated autophagy (23). To explore the biological significance of miR-204, we stably overexpressed miR-204 in two RCC cell lines OSRC-2 and 786-O with lentiviruses carrying miR-204 and its control (miR-NC). The efficacy of infection was tested by qRT-PCR (Fig. 2A). Overexpression of miR-204 markedly reduced RCC cell proliferation compared with miR-NC in the OSRC-2 and 786-O cell lines (Fig. 2B and C). Similarly, miR-204 overexpression suppressed cell invasion in the two tested RCC cell lines (Fig. 2D and E).

Conversely, we examine the phenotype in both cell lines with anti-miR-204. RT-PCR indicated that anti-miR-204 was effective when we treated the cells with miR-204 inhibitor (Fig. 2F). MTT proliferation and Transwell invasion assays showed that anti-miR-204 significantly increased the growth and invasive capability of both RCC cell lines compared with the anti-miR-NC group (Fig. 2G-J).

Taken together, these findings indicate that miR-204 acts as a tumor suppressor in RCC by inhibiting cell proliferation and invasion.

miR-204 suppresses $R C C$ cell proliferation and invasion in vivo. To identify the antitumorigenic roles of miR-204 in RCC in vivo, OSRC-2 cells overexpressing miR-204 or cells transfected with miR-NC were injected into the left kidney of nude mice. Tumor growth and metastasis were then assessed by detecting GFP expression using an automated fluorescence imaging system. As shown in Fig. 3A, fluorescence imaging showed a marked reduction in tumor growth in the miR-204-overexpressing cells as early as week 3 , as revealed by the determination of tumor mass (Fig. 3B). Consistently, the tumor volume measurement also confirmed the conclusion that overexpression of miR-204 inhibited tumorigenicity of the OSRC-2 cells (Fig. 3C).

In addition, no macroscopic metastases were found in the tumors with miR-204 overexpression while the miR-NC tumors were prone to local invasion and metastasis through the automated fluorescence imaging system (Fig. 3D). As shown in Fig. 3E, the miR-204 tumors derived from the OSRC-2 cells metastasized to the lung less frequently than the miR-NC tumors. These data above further demonstrated that miR-204 functions as a critical tumor suppressor in RCCs by suppressing tumorigenesis and metastatic colonization. 
A

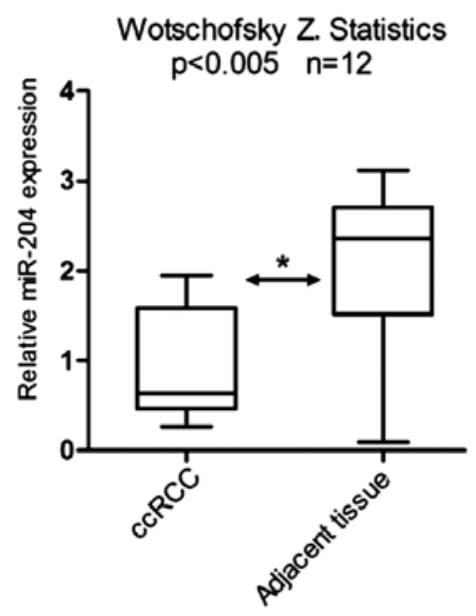

C

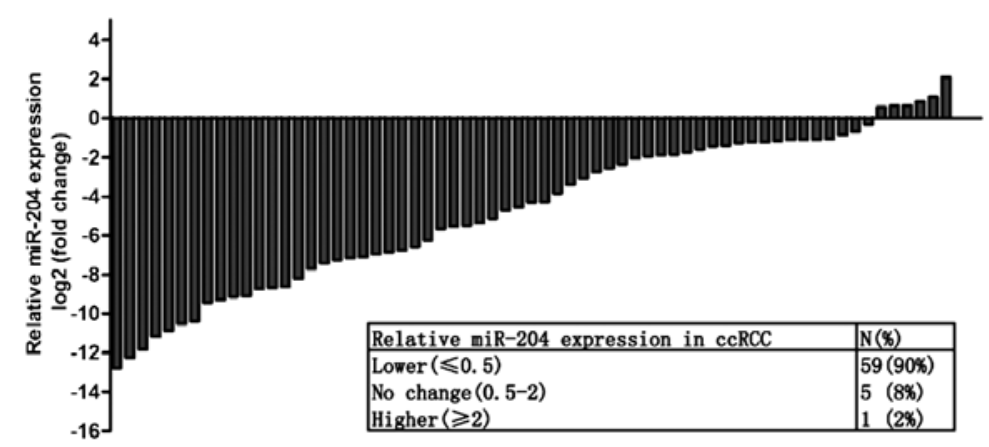

B

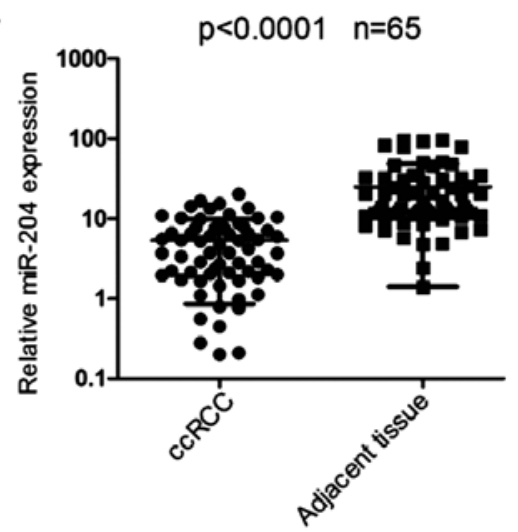

D

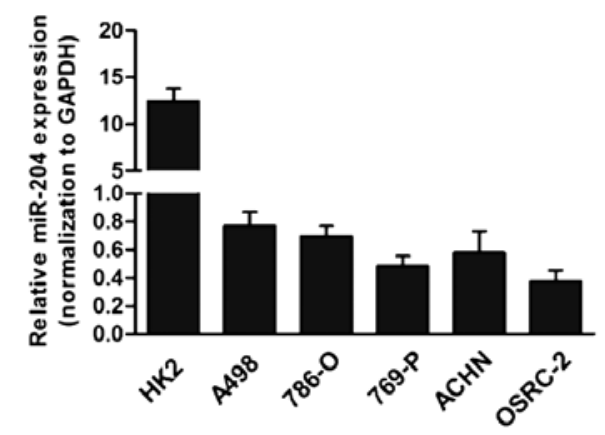

Figure 1. miR-204 expression is downregulated in RCC tissues and cell lines. (A) Analysis of an RCC array, using NCBI GEO dataset of the statistical results of Wotschofsky et al (GSE37989) (24), showing the miR-204 level in 12 RCC samples. (B) miR-204 expression in human RCC tissues and paired adjacent non-cancerous renal tissues. (C) Relative miR-204 expression levels in RCC tumors are presented as fold-change $=2$ ( $\Delta \mathrm{Ct}$ normal - $\Delta \mathrm{Ct}$ tumor) of tumor vs. matched normal tissues. The 0.5 fold-change threshold was defined as differentially expressed. The relative miR-204 expression levels in RCC samples are summarized in Table II. (D) miR-204 expression in a series of RCC cell lines or immortalized proximal tubule epithelial cells from normal adult human kidney (HK2, A498, 786-O, 769-P, ACHN and OSRC-2). For B-D, the expression level of miR-204 was analyzed by qRT-PCR. The horizontal lines in the box plots represent the medians, the boxes represent the interquartile range, and the whiskers represent the 2.0 and 97.5 th percentiles. The significant differences between samples were analyzed using the Wilcoxon signed-rank test.

miR-204 directly targets $R A B 22 A$. To dissect the potential downstream gene which is targeted by miR-204 in RCC cells, we predicted the potential miR-204 targets using three bioinformatic analysis softwares (TargetScan, miRanda and miRDB) (Fig. 4A), and explored the common 10 downregulated transcripts and potential targets of miR-204 (HOXA10, TGFBR2, RUNX2, MMP9, SIRTUIN1, FOXC1, IL11, RAB22A, EZRIN and MEIS1). To validate these 10 candidates, we firstly introduced miR-204 overexpression lentiviruses into the OSRC-2 cell line. We next applied the RT-PCR assay to validate the 11 predicted miRNA target genes in the OSRC-2 cells with overexpression of miR-204. We found 6 candidate genes (HOXA10, RUNX2, MMP9, FOXC1, IL11 and RAB22A) that were downregulated by overexpression of miR-204 in the OSRC-2 miR-204-transfected cells (Fig. 4B). We then used another RCC 786-O cell line to further identify the candidate genes. Furthermore, RT-PCR analysis showed that miR-204 led to a decrease in RAB22A expression in the 786-O cell line (Fig. 4C).

To further examine RAB22A as a direct target gene of miR-204, OSRC-2 and 786-O cells were transfected with miR-204 overexpression lentiviruses, respectively. The protein level of RAB22A was substantially decreased after ectopic miR-204 transfection in both cell lines (Fig. 4D and E).
Based on TargetScan prediction analysis, 3 miR-204-targeting sites were identified in the 3'-UTR of RAB22A mRNA (Fig. 4F), and mutant vectors of RAB22A 3'-UTR containing four mutated bases on the predicted binding sites were constructed (MUT-1, MUT-2, MUT-3 and MUT-1-3). Transient transfection of OSRC-2 cells with the above wild-type or mutant vectors and miR-204 or mock control resulted in partial rescue of the inhibition (Fig. 4G).

Immunohistochemical staining was carried out to assess RAB22A expression in the ccRCC tissues. Higher expression was noted in the tissues than the level in the adjacent normal tissues (Fig. 4H). miR-204 expression was negatively related to RAB22A protein expression in the ccRCC samples (Fig. 4I). Together, results from Fig. 4 demonstrated that RAB22A is a direct target of miR-204 and was inversely correlated with the endogenous miR-204 expression in primary ccRCC tissues.

miR-204-suppressed tumor proliferation and invasion are modulated by $R A B 22 A$. The findings above indicate that RAB22A is a candidate effector to mediate the biological functions of miR-204. We then identified whether overexpression of RAB22A (oe-RAB22A) could recapitulate the inhibitory effects of miR-204 on RCC cell proliferation and invasion. OSRC-2 and 786-O cells were transfected with oe-RAB22A 
A

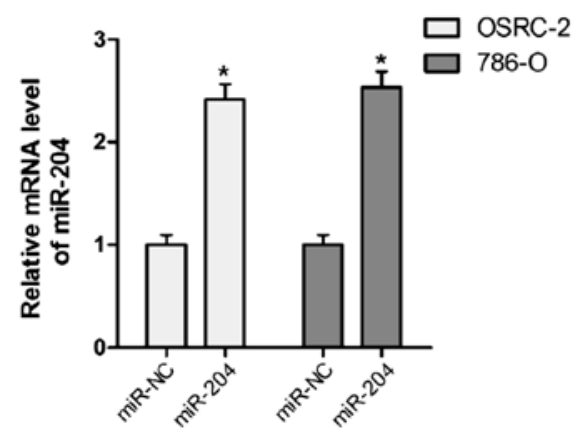

C

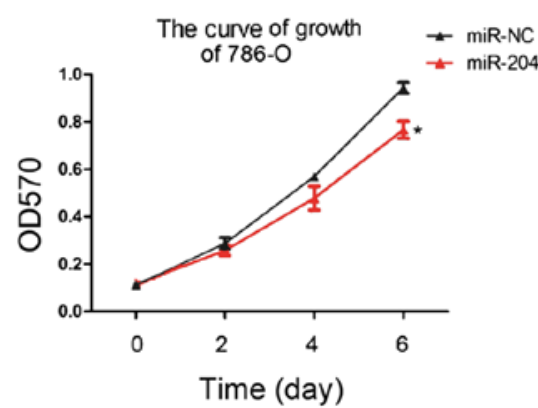

B

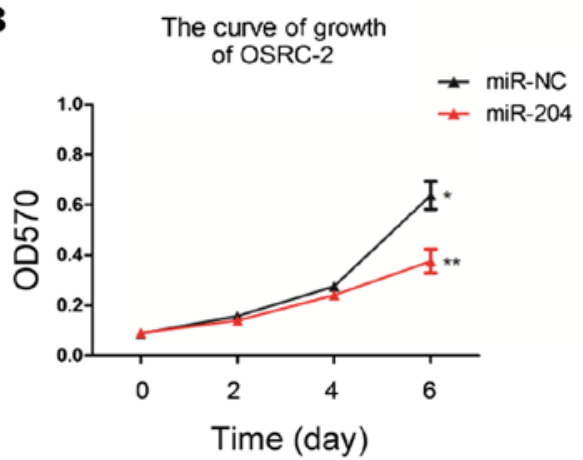

D

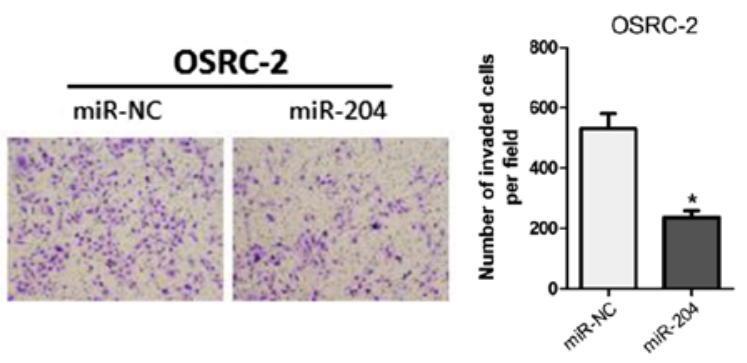

$\mathbf{E}$


F

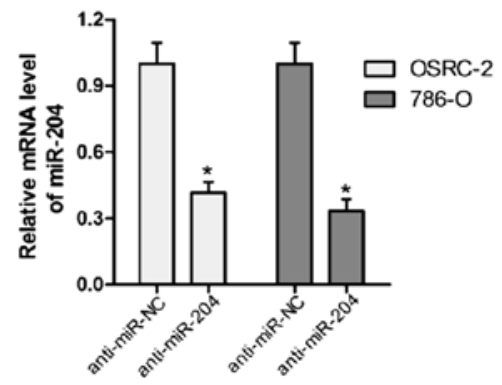

G

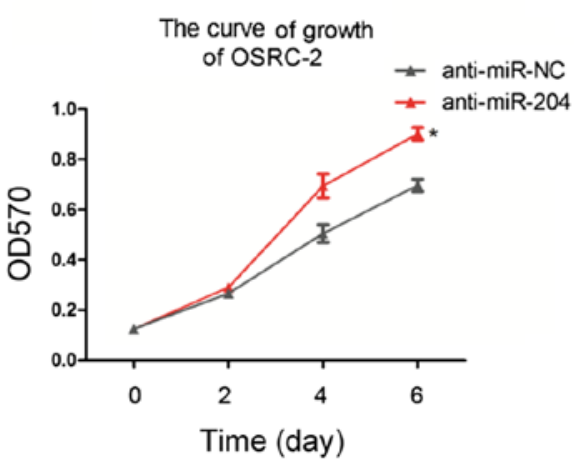

H

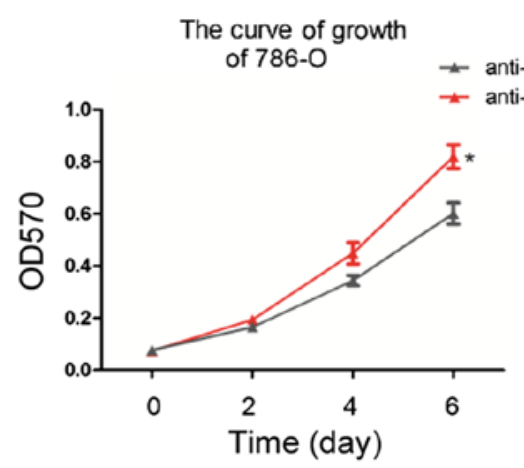

I

OSRC-2

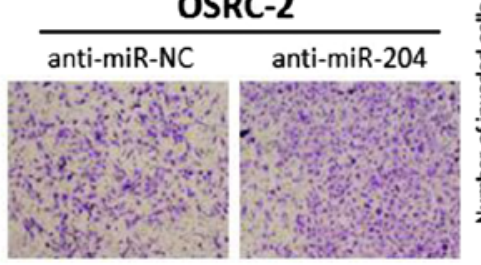

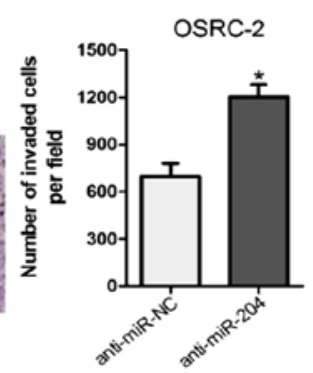

$\mathbf{J}$
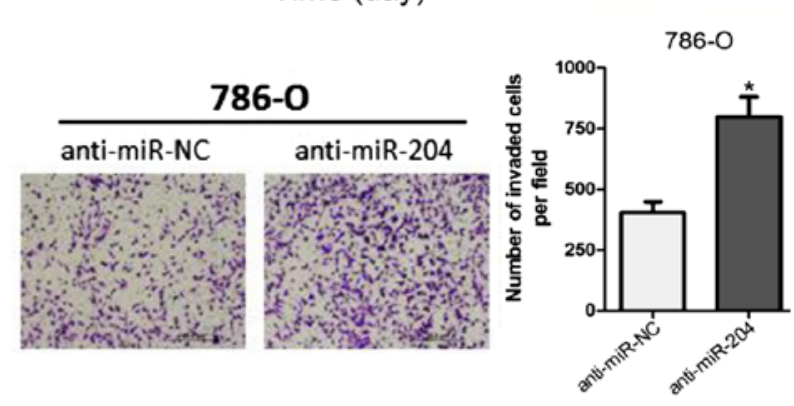

Figure 2. miR-204 attenuates RCC cell proliferation and invasion in vitro. (A) Real-time RT-PCR assays of the miR-204 level in stable OSRC-2 and 786-O cell clones. The change in proliferation as determined by MTT assays following transfection of miR-204 compared with miR-NC in the (B) OSRC-2 and (C) 786-O cells. Experiments in B and $\mathrm{C}$ were repeated at least 3 times with similar results, and error bars represent mean \pm SD. (D and E) Representative images (left panels). The number of invasive cells per high-power field (right panels) was reduced following transfection of miR-204 in the (E) OSRC-2 and (F) 786-O cells when compared with that of the cells transfected with miR-NC. (F) Real-time RT-PCR assays of the miR-204 level in anti-miR-204 RCC cell clones. The change in proliferation as determined by MTT assay following transfection of anti-miR-204 compared with anti-miR-NC in the (G) OSRC-2 and (H) 786-O cells. (I and J) Representative images (left panels) of the invasive cells. The number of invasive cells per high-power field (right panel) was reduced by transfection of anti-miR-204 in the (I) OSRC-2 and (J) 786-O cells when compared with that of the anti-miR-NC cells. ${ }^{*} \mathrm{P}<0.05$; ${ }^{* *} \mathrm{P}<0.01$. 
A

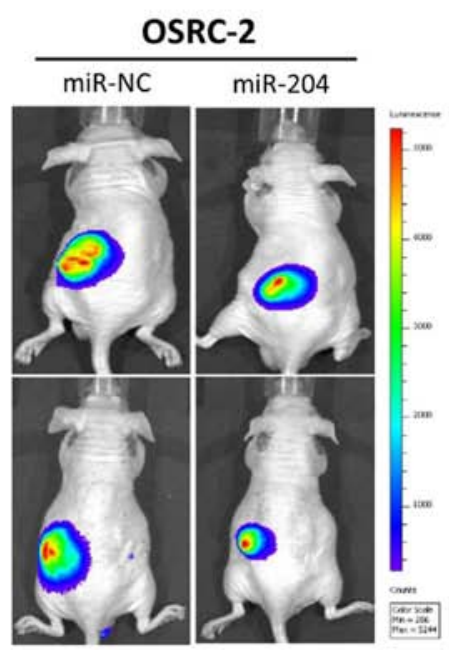

C



E

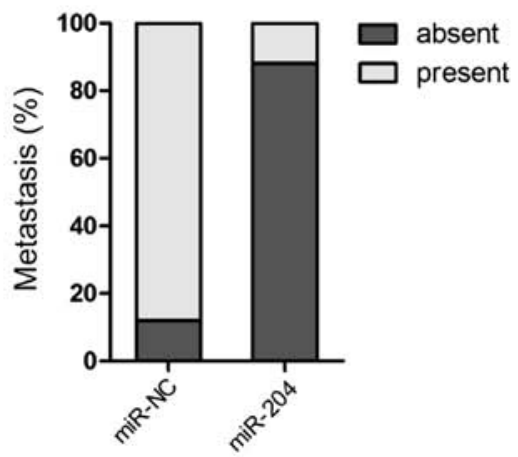

B

miR-NC

miR-204

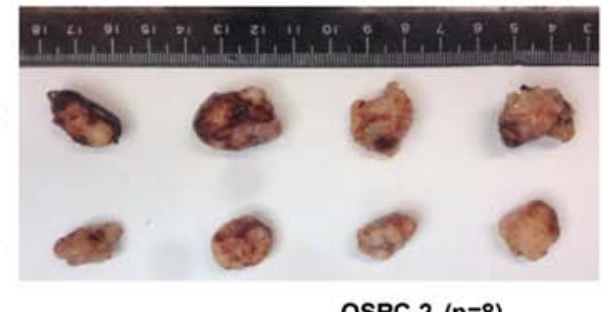

OSRC-2 $(n=8)$

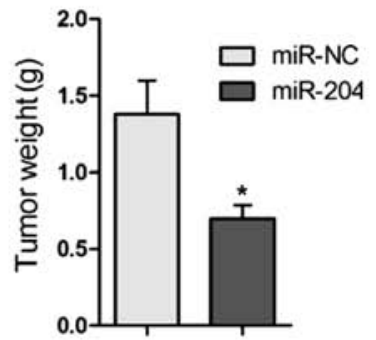

D

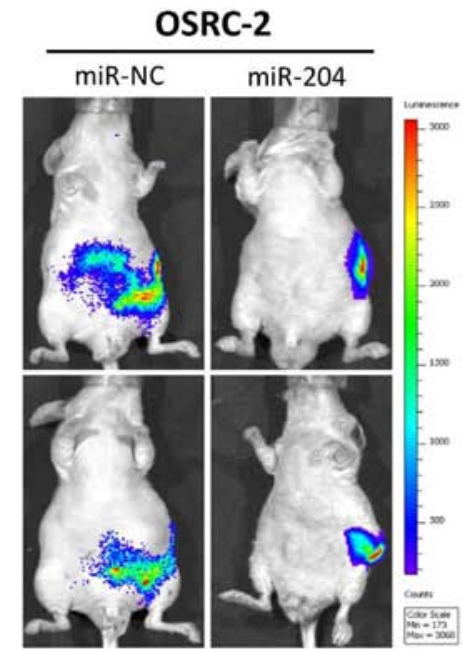

Figure 3. miR-204 suppresses RCC cell proliferation and invasion in vivo. (A) Representative images of mice viewed by the IVIS Lumina II system in the miR-204 and miR-NC groups 4 weeks after left renal capsule injection $(\mathrm{n}=8)$. (B) Macroscopic appearance of the tumor xenografts in nude mice from the 7- to 8 -week group following OSRC-2 cell injection. Weights of the xenografts are shown (mean $\pm \mathrm{SD})(\mathrm{n}=8)$. (C) Tumor volumes were evaluated periodically at the indicated days post-inoculation of OSRC-2 cells. Tumor volumes were calculated as width ${ }^{2} \mathrm{x}$ length $\mathrm{x} 0.5\left(\mathrm{~mm}^{3}\right)$ every 7-8 days. Data represent mean \pm SD. (D) Orthotopic xenograft animal models were also generated using miR-204 and miR-NC cells. Presented are representative images of abdominal metastasis viewed by the IVIS Lumina II system in each group 4 weeks after orthotopic xenograft transplantation (n=8). (E) OSRC-2 cells stably transfected with miR-NC metastasized to multiple organs. ${ }^{*} \mathrm{P}<0.05 ;{ }^{* *} \mathrm{P}<0.01$.

vs. oe-mock. Significantly, oe-RAB22A in RCC cells induced cell growth (Fig. 5A and B), similarly to the phenotypic alterations upon miR-204 overexpression. In addition, oe-RAB22A led to enhance cell invasion in the OSRC-2 and 786-O cells (Fig. 5C and D).

To further determine whether RAB22A is a direct and functional mediator of miR-204-suppressed cell invasion, we performed a rescue experiment by co-transfection of oe-RAB22A (vs. mock) and oe-miR-204 (vs. miR-NC) into the OSRC-2 and 786-O cell lines. The enhancement in RCC cell invasion reduced by miR-204 overexpression was effectively reversed by oe-RAB22A (Fig. 5E and F). Collectively, these findings indicate that RAB22A is an essential functional effector of miR-204 in RCC.

\section{Discussion}

Recent studies have found that miRNAs play a fundamental role in the proliferation, invasion and metastasis of malignant human cancers (25), involving renal cell carcinoma 
A

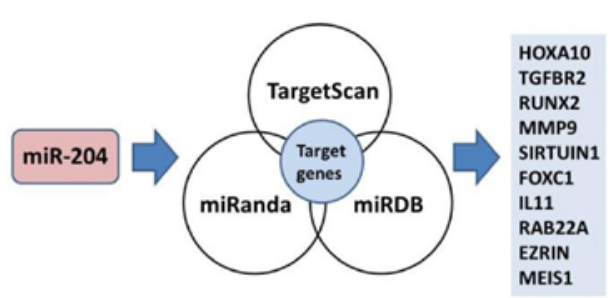

C

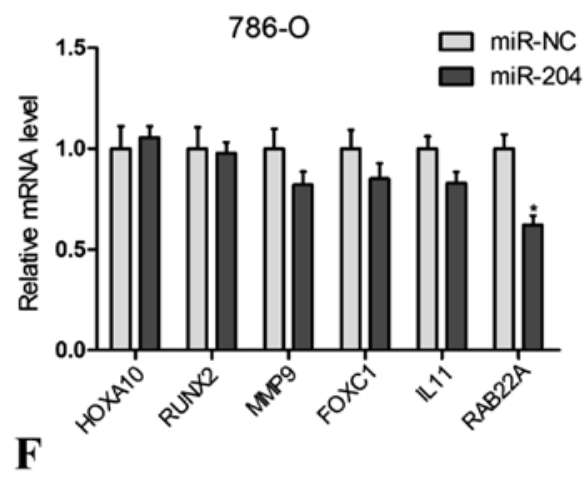

B

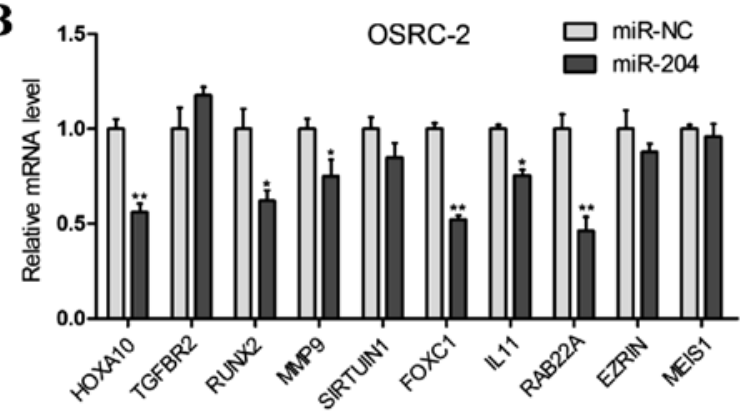

D

\section{E}
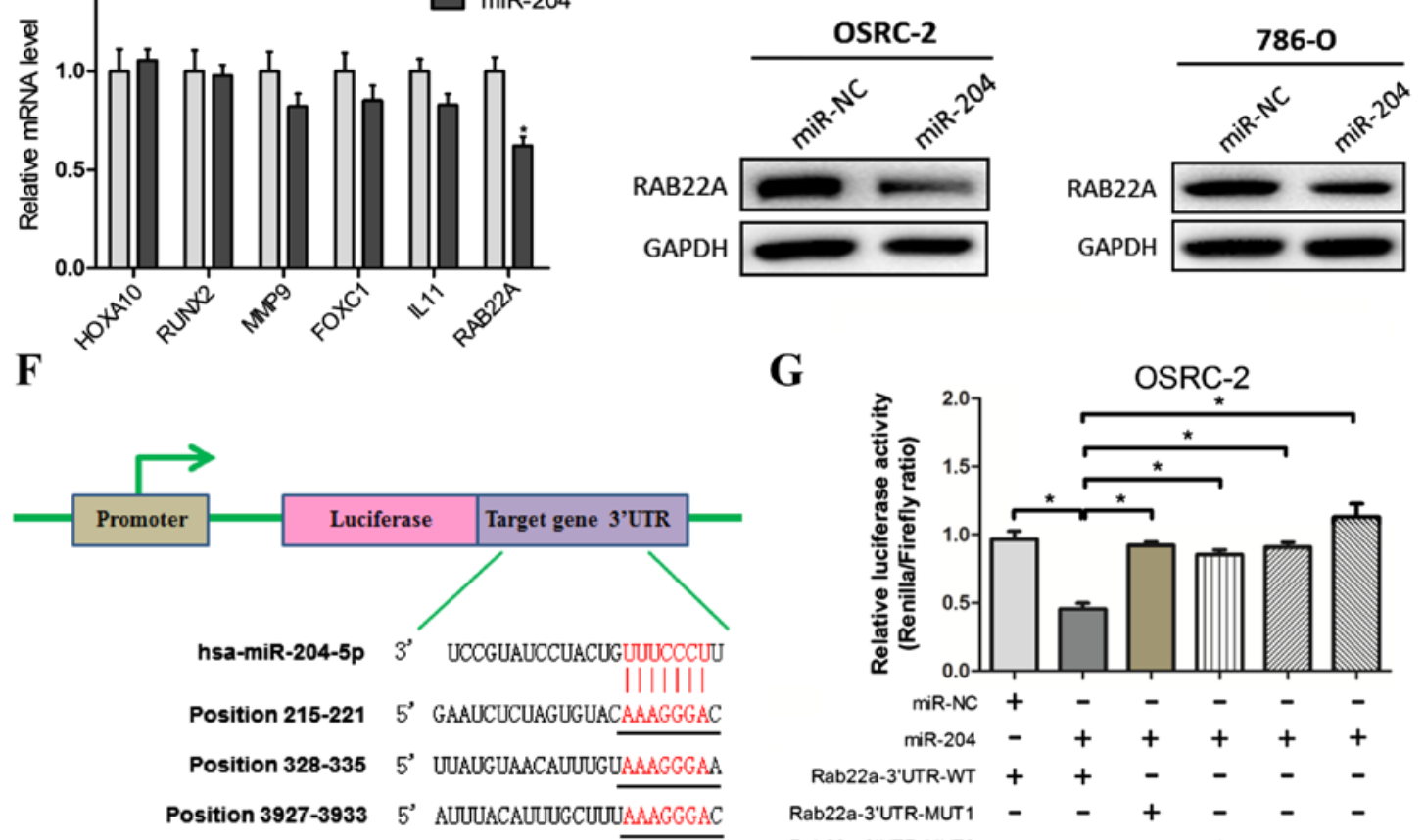

G

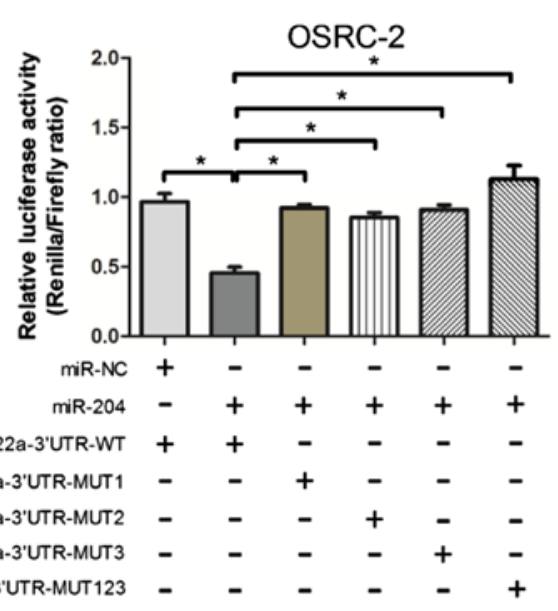

H

RAB22A
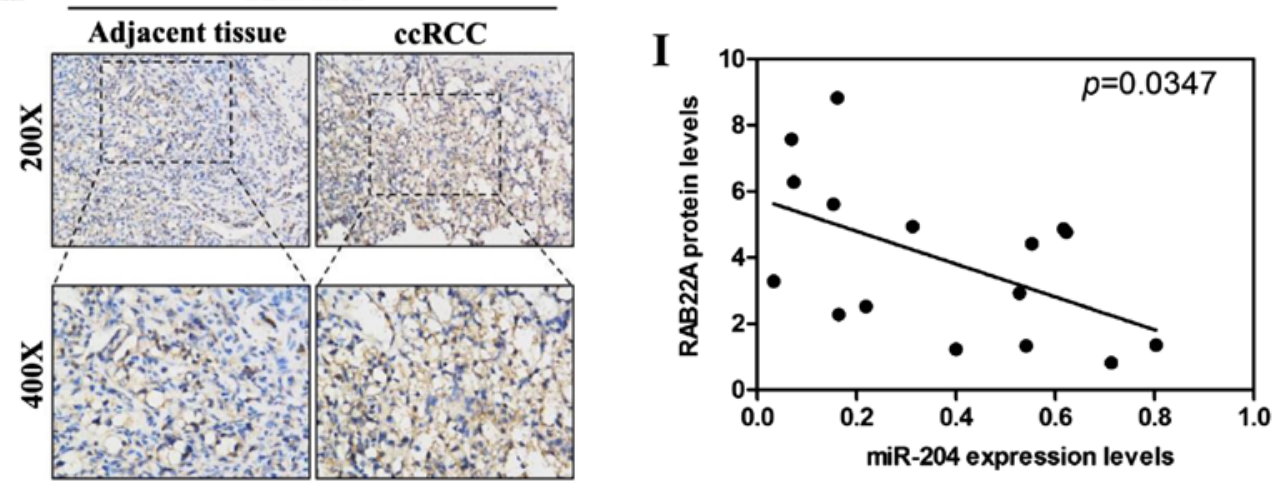

Figure 4. miR-204 directly targets RAB22A. (A) A schematic illustration of the proposed model depicting 10 putative target genes of miR-204 as mentioned above from 3 microRNA prediction databases: targetscan.org, mirdb.org and miranda.org. (B) Real-time PCR of 10 putative target genes of miR-204 relative to U6 expression for miR-204 in the OSRC-2 cells. (C) Real-time PCR of 6 putative target genes of miR-204 relative to U6 expression for miR-204 in the 786-O cells. Western blot analyses were performed to examine the effects of miR-204 on RAB22A gene expression in the (D) OSRC-2 and (E) 786-O cells. (F) Putative miR-204 binding sequence in the 3'-UTR of RAB22A mRNA. (G) Relative activity of the luciferase gene fused with the wild-type or mutant 3'-UTR of RAB22A. * P<0.05. (H) Immunohistochemical staining indicating the location of RAB22A expression in the ccRCC specimens (magnification, $\mathrm{x} 200$ and $\mathrm{x} 400$ ). (I) Correlation between miR-204 expression and $\mathrm{RAB} 22 \mathrm{~A}$ protein levels in the ccRCC tissues. ${ }^{*} \mathrm{P}<0.05$; ${ }^{* *} \mathrm{P}<0.01$.

(RCC) (26). Previous studies indicate that miR-204 is downregulated in various human tumors and acts as a tumor suppressor negatively correlated with tumorigenesis and progression, including endometrial and gastric cancer, and nasopharyngeal carcinoma (27-29). In the present study, we found that the expression of miR-204 in RCC tissues and cell 

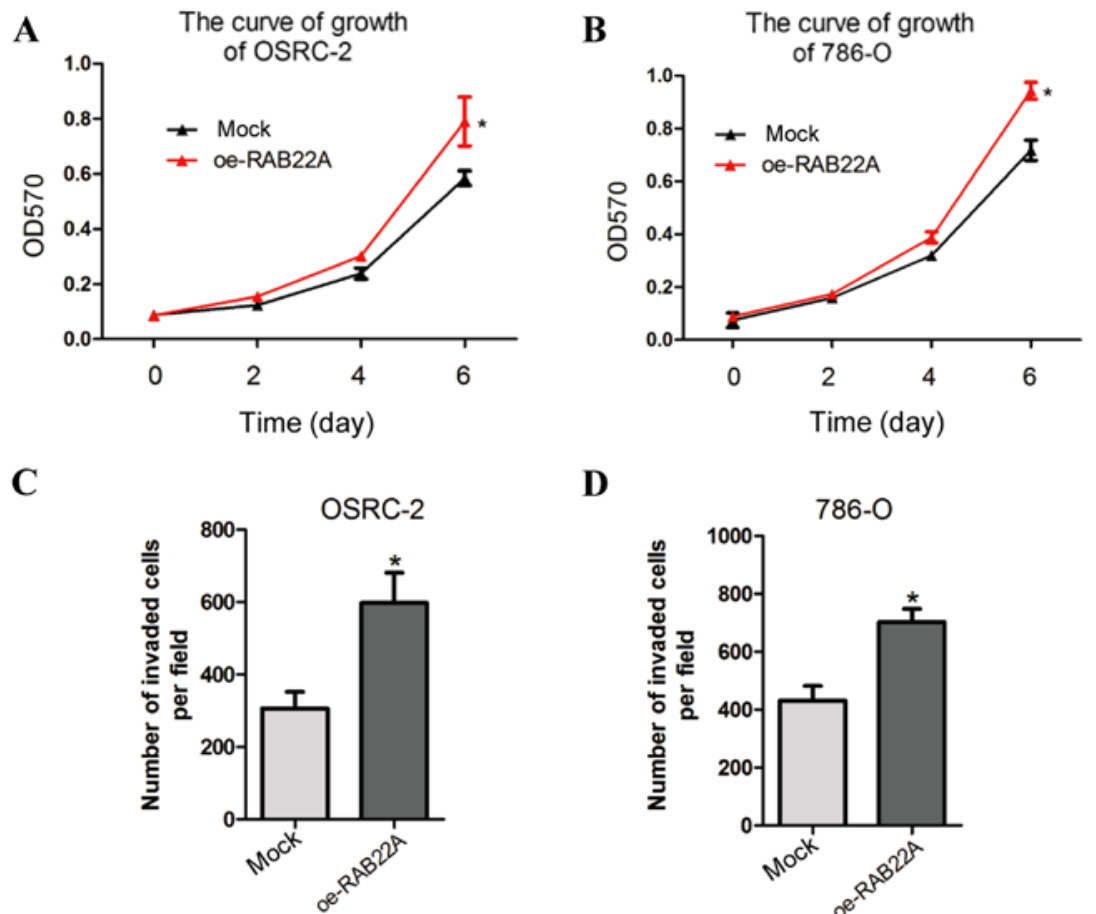

D

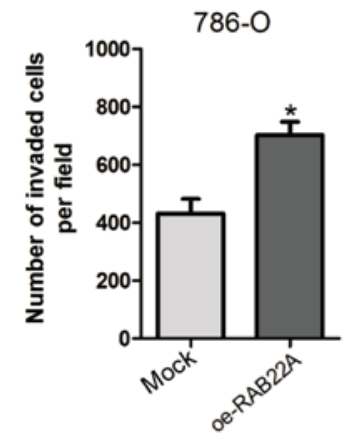

$\mathbf{E}$

OSRC-2

$\mathbf{F}$
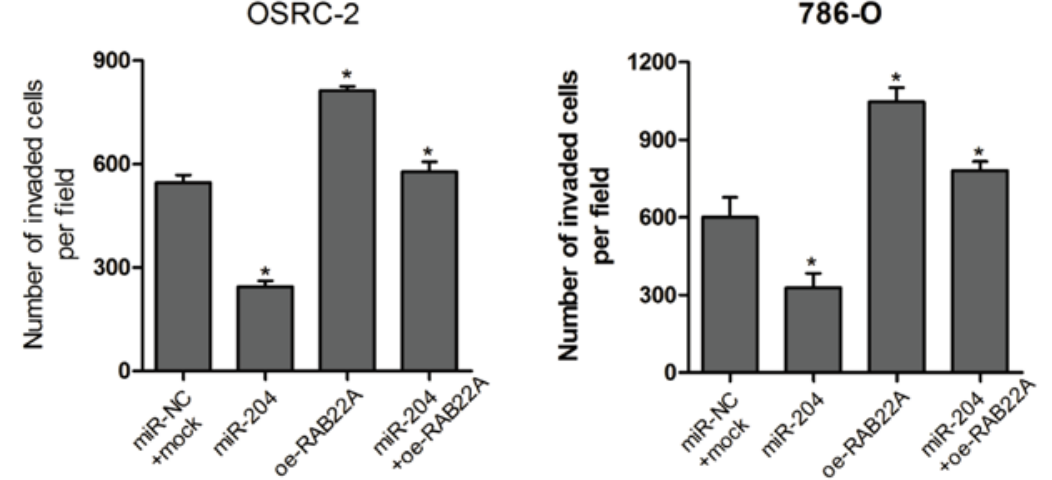

Figure 5. miR-204-reduced tumor proliferation and invasion is modulated by RAB22A. (A) Change in proliferation as determined by the MTT assay following transfection of oe-RAB22A compared with mock in the (A) OSRC-2 and (B) 786-O cells. Experiments in A and B were repeated at least 3 times with similar results, and error bars represent the mean $\pm \mathrm{SD}$. ${ }^{*} \mathrm{P}<0.05$. (C) Number of invasive cells per high-power field was reduced following transfection of oe-RAB22A in the (C) OSRC-2 and (D) 786-O cells when compared with that of the mock cells. Cell invasion in (E) OSRC-2 and (F) 786-O cells was analyzed by Transwell assays. Cells were co-transfected with oe-RAB22A or mock and miR-204 or miR-NC. Data shown are mean $\pm \mathrm{SD}(\mathrm{n}=3)$; ${ }^{*} \mathrm{P}<0.05$.

lines was downregulated compared to that in surrounding normal tissues and normal HK2 cell lines, similar to a study by Mikhaylova et al (23).

Next, results from in vitro and in vivo experiments demonstrated that miR-204 markedly suppressed the growth and invasion of RCC cell lines OSRC-2 and 786-O. Furthermore, we noted that the lower miR-204 levels were associated with promotion of RCC proliferation and invasion. Taken together, all these data strongly support the conclusion that miR-204 can be a potential biomarker in RCC progression by inhibition of cell proliferation and invasion.

A previous study demonstrated that RAB22A is a direct target of several miRNAs. Zhang et al found that miR-373 targeting of the Rab22a oncogene suppressed tumor invasion and metastasis in ovarian cancer (30). miR-203 has been reported as a tumor-suppressor gene in osteosarcoma by regulating $\mathrm{RAB} 22 \mathrm{~A}$ (31). The present study identified that miR-204 inhibited RAB22A expression by directly targeting 3'UTR of RAB22A in RCC. Overexpression of miR-204 in RCC cell lines led to decreased expression of RAB22A, and then suppressed RCC proliferation and invasion. The interruption approach using $\mathrm{RAB} 22 \mathrm{~A}$ overexpression reversed the oe-miR-204 effects on RCC OSRC-2 and 786-O cell proliferation and invasion. In contrast, knockdown of miR-204 resulted in increased RAB22A expression and enhanced cell growth and invasive capability in RCC. More importantly, the expression of miR-204 was negatively correlated with the RAB22A expression level in RCC tissues.

RAB22A, acting as a small GTPase, serves as a member of the RAB family endocytic pathway (32). Several studies have shown that RAB22A plays an oncogenic role in liver cancer and ovarian cancer $(30,33)$. The explored mechanism 
indicates that RAB22A is mainly associated with EMT signaling to modulate cancer cell progression. In addition, hypoxia-induced HIF-dependent RAB22A expression is associated with breast cancer patient mortality (34). However, the role of RAB22A in RCC was not well established. Based on our findings, overexpression of RAB22A promoted cell proliferation and invasion in OSRC-2 and 786-O RCC cell lines. In conclusion, data presented in the present study provided a firm foundation that RAB22A holds great promise as a therapeutic target in RCC.

In summary, we demonstrated the marked alteration of miR-204 expression in human RCC tissues and cell lines, and we found that ectopic expression of miR-204 could efficiently suppress cell proliferation and invasion of RCC cells in vitro and in vivo by directly targeting RAB22A 3 '-UTR. These specific functional mediators may provide novel mechanisms in tumor progression and additional diagnostic and/or therapeutic targets. The present study extends our knowledge that the specification of the miR-204-RAB22A pathway also has fundamental importance in RCC progression.

\section{Acknowledgements}

The authors thank the patients and clinicians for their contributions to the present study.

\section{References}

1. Compérat E and Camparo P: Histological classification of malignant renal tumours at a time of major diagnostic and therapeutic changes. Diagn Interv Imaging 93: 221-231, 2012.

2. Ramana J: RCDB: Renal Cancer Gene Database. BMC Res Notes 5: 246, 2012.

3. Gupta K, Miller JD, Li JZ, Russell MW and Charbonneau C: Epidemiologic and socioeconomic burden of metastatic renal cell carcinoma (mRCC): A literature review. Cancer Treat Rev 34: 193-205, 2008.

4. Stadler WM: Targeted agents for the treatment of advanced renal cell carcinoma. Cancer 104: 2323-2333, 2005.

5. Polyzos A: Activity of SU11248, a multitargeted inhibitor of vascular endothelial growth factor receptor and platelet-derived growth factor receptor, in patients with metastatic renal cell carcinoma and various other solid tumors. J Steroid Biochem Mol Biol 108: 261-266, 2008

6. Atkins MB, Ernstoff MS, Figlin RA, Flaherty KT, George DJ, Kaelin WG Jr, Kwon ED, Libermann TA, Linehan WM, McDermott DF, et al: Innovations and challenges in renal cell carcinoma: Summary statement from the Second Cambridge Conference. Clin Cancer Res 13: 667s-670s, 2007.

7. Longo R, D'Andrea MR, Sarmiento R, Salerno F and Gasparini G: Integrated therapy of kidney cancer. Ann Oncol 18 (Suppl 6): vi141-vi148, 2007.

8. Bagga S, Bracht J, Hunter S, Massirer K, Holtz J, Eachus R and Pasquinelli AE: Regulation by let -7 and lin- 4 miRNAs results in target mRNA degradation. Cell 122: 553-563, 2005.

9. Stallings RL: MicroRNA involvement in the pathogenesis of neuroblastoma: Potential for microRNA mediated therapeutics. Curr Pharm Des 15: 456-462, 2009.

10. Jung M, Mollenkopf HJ, Grimm C, Wagner I, Albrecht M, Waller T, Pilarsky C, Johannsen M, Stephan C, Lehrach H, et al: MicroRNA profiling of clear cell renal cell cancer identifies a robust signature to define renal malignancy. J Cell Mol Med 13: 3918-3928, 2009.

11. Majid S, Saini S, Dar AA, Hirata H, Shahryari V, Tanaka Y, Yamamura S, Ueno K, Zaman MS, Singh K, et al: MicroRNA-205 inhibits Src-mediated oncogenic pathways in renal cancer. Cancer Res 71: 2611-2621, 2011.

12. Hirata H, Hinoda $Y$, Ueno K, Nakajima K, Ishii N and Dahiya R: MicroRNA-1826 directly targets beta-catenin (CTNNB1) and MEK1 (MAP2K1) in VHL-inactivated renal cancer. Carcinogenesis 33: 501-508, 2012.
13. Doberstein K, Steinmeyer N, Hartmetz AK, Eberhardt W, Mittelbronn M, Harter PN, Juengel E, Blaheta R, Pfeilschifter J and Gutwein P: MicroRNA-145 targets the metalloprotease ADAM17 and is suppressed in renal cell carcinoma patients. Neoplasia 15: 218-230, 2013.

14. Schickel R, Park SM, Murmann AE and Peter ME: miR-200c regulates induction of apoptosis through CD95 by targeting FAP-1. Mol Cell 38: 908-915, 2010.

15. Saini S, Yamamura S, Majid S, Shahryari V, Hirata H, Tanaka Y and Dahiya R: MicroRNA-708 induces apoptosis and suppresses tumorigenicity in renal cancer cells. Cancer Res 71: 6208-6219, 2011.

16. Koo S, Martin GS, Schulz KJ, Ronck M and Toussaint LG: Serial selection for invasiveness increases expression of miR-143/ miR-145 in glioblastoma cell lines. BMC Cancer 12: 143, 2012.

17. Ma F, Zhang J, Zhong L, Wang L, Liu Y, Wang Y, Peng L and Guo B: Upregulated microRNA-301a in breast cancer promotes tumor metastasis by targeting PTEN and activating Wnt/ $\beta$ catenin signaling. Gene 535: 191-197, 2014.

18. Lu L, Xue X, Lan J, Gao Y, Xiong Z, Zhang H, Jiang W, Song W and Zhi Q: MicroRNA-29a upregulates MMP2 in oral squamous cell carcinoma to promote cancer invasion and anti-apoptosis. Biomed Pharmacother 68: 13-19, 2014.

19. Landgraf P, Rusu M, Sheridan R, Sewer A, Iovino N, Aravin A, Pfeffer S, Rice A, Kamphorst AO, Landthaler M, et al: A mammalian microRNA expression atlas based on small RNA library sequencing. Cell 129: 1401-1414, 2007.

20. Lee Y, Yang X, Huang Y, Fan H, Zhang Q, Wu Y, Li J, Hasina R, Cheng C, Lingen MW, et al: Network modeling identifies molecular functions targeted by miR-204 to suppress head and neck tumor metastasis. PLOS Comput Biol 6: e1000730, 2010.

21. Sacconi A, Biagioni F, Canu V, Mori F, Di Benedetto A, Lorenzon L, Ercolani C, Di Agostino S, Cambria AM, Germoni S, et al: miR-204 targets Bcl-2 expression and enhances responsiveness of gastric cancer. Cell Death Dis 3: e423, 2012.

22. Chen Z, Sangwan V, Banerjee S, Mackenzie T, Dudeja V, Li X, Wang H, Vickers SM and Saluja AK: miR-204 mediated loss of Myeloid cell leukemia-1 results in pancreatic cancer cell death. Mol Cancer 12: 105, 2013.

23. Mikhaylova O, Stratton Y, Hall D, Kellner E, Ehmer B, Drew AF, Gallo CA, Plas DR, Biesiada J, Meller J, et al: VHL-regulated miR-204 suppresses tumor growth through inhibition of LC3Bmediated autophagy in renal clear cell carcinoma. Cancer Cell 21: 532-546, 2012.

24. Wotschofsky Z, Liep J, Meyer HA, Jung M, Wagner I, Disch AC, Schaser KD, Melcher I, Kilic E, Busch J, et al: Identification of metastamirs as metastasis-associated microRNAs in clear cell renal cell carcinomas. Int J Biol Sci 8: 1363-1374, 2012.

25. Baranwal S and Alahari SK: miRNA control of tumor cell invasion and metastasis. Int J Cancer 126: 1283-1290, 2010.

26. Petillo D, Kort EJ, Anema J, Furge KA, Yang XJ and Teh BT: MicroRNA profiling of human kidney cancer subtypes. Int $J$ Oncol 35: 109-114, 2009.

27. Chung TK, Lau TS, Cheung TH, Yim SF, Lo KW, Siu NS, Chan LK, Yu MY, Kwong J, Doran G, et al: Dysregulation of microRNA-204 mediates migration and invasion of endometrial cancer by regulating FOXC1. Int J Cancer 130: 1036-1045, 2012.

28. Zhou X, Li L, Su J and Zhang G: Decreased miR-204 in H. pyloriassociated gastric cancer promotes cancer cell proliferation and invasion by targeting SOX4. PLoS One 9: e101457, 2014.

29. Ma L, Deng X, Wu M, Zhang G and Huang J: Down-regulation of miRNA-204 by LMP-1 enhances CDC42 activity and facilitates invasion of EBV-associated nasopharyngeal carcinoma cells. FEBS Lett 588: 1562-1570, 2014.

30. Zhang Y, Zhao FJ, Chen LL, Wang LQ, Nephew KP, Wu YL and Zhang S: MiR-373 targeting of the Rab22a oncogene suppresses tumor invasion and metastasis in ovarian cancer. Oncotarget 5: 12291-12303, 2014.

31. Yang D, Liu G and Wang K: miR-203 acts as a tumor suppressor gene in osteosarcoma by regulating RAB22A. PLoS One 10: e0132225, 2015.

32. Mesa R, Salomón C, Roggero M, Stahl PD and Mayorga LS: Rab22a affects the morphology and function of the endocytic pathway. J Cell Sci 114: 4041-4049, 2001.

33. He H, Dai F, Yu L, She X, Zhao Y, Jiang J, Chen X and Zhao S: Identification and characterization of nine novel human small GTPases showing variable expressions in liver cancer tissues. Gene Expr 10: 231-242, 2002.

34. Wang T, Gilkes DM, Takano N, Xiang L, Luo W, Bishop CJ, Chaturvedi P, Green JJ and Semenza GL: Hypoxia-inducible factors and RAB22A mediate formation of microvesicles that stimulate breast cancer invasion and metastasis. Proc Natl Acad Sci USA 111: E3234-E3242, 2014. 